\title{
Los Sistemas de Recuperación de Información por indexación de triadas, una experiencia
}

\author{
Gerardo Vega Hernández \\ Inves ti ga dor Di rec ción Ge ne ral de Servi cios de \\ Cóm pu to Aac dé mi co, UnA M \\ Circuito Exterior, CiudadUniversitaria \\ Te1.56-22--85-09 \\ E/Mail:gerardov@servidor.unam.mx
}

\section{RESUMEN}

$E$ s frecuen te que los Sis te mas de Recu pe ra ción de In for ma ción que rea li zan in de xa ción au tomá ti ca, uti li cen las pa la bras de un do cu men to para di fe ren ciae este del res to de la colec ción de do cu men tos. Esto trae como con se cuen cia la ne ce si dad de idear, en mu chos ca sos, com plejos alforitmos que sean ca pa ces de "com pren der" la mor fo lo gía del idio ma en que esté es crita la colec ción de docu men tos. En el presen te ar tícu lose des criben los por me no res y vi ven cias que se han te ni do en la in ves ti ga ción y de sa rrollo de un sis te ma de Recu pe ra ción de In formación que realiza la indexación au to má ti ca por tria das de le tras en lu gar de pa la bras.

\section{A BSTRACT}

A utomatic Indexation Information Retrieval Systems often use words from a document to diffe ren tia te the lat ter from the re maining docu ment collection, thus lea ding in many cases to the need of con tri ving com plex al go rithms ca pa ble of "un ders tan ding" the mor pho logy of the lan gua ge in which the docu ment collec tion has been written. This ar tic le des cribes de tails and ex pe rien ces in the re se rach and de ve lopment of a Re trie val In for ma tion Sys tem that carries out automaticalindexation by means of letter-triads, as opposed to words.

\section{INTRODUCCIÓN}

$\mathrm{E}$ n la actualidad se genran todos los días gran des can ti da des de in for ma ción es crita. Sim ple men te en la UNAM exis te una gran va rie dad de ban cos de in for ma ción que se actua li zan prác tica men te to dos los días. Por esta ra zón es in ne ga ble la ne ce si dad de con tar con sistemasautomatizados de consulta de información que permitan la búsqueda en texto completo. Existen a este respecto, Sistemas de Recuperación de Información en texto completo (los que serán abrevidados en lo subsecuentesimplementecomo SRI), capaces de representar, almacenar, organizar y accesar la in for ma ción dis po ni ble a tra vés de una computadora. En otras palabras, estos sistemas son el medio por el cual un usuario en buscade in for mación, ac cesa eficien te men te la o las co lec ciones de do cu men tos de su in terés que de al gu na for ma se en cuen tran al macenados en una computadora.
Los SRI pueden ser divididos en dos categorías: lo que em plean ín di ces para la bús que da de la información y los que no los utilizan. Los últimos realizan las búsquedas sobre el texto original y generalmente están basados en algoritmos rápidos de búsqueda de cadenas de carac teres. ${ }^{(1-3)}$ Este tipo de al go tir mos se emplea comúnmente para el desarrollo de ru ti nas de bús que da para los edi to res de tex to y manuales en línea. Por otro lado, los sistemas que utilizaníndices en las búsquedas, se caracterizan por no utilizar directamente los textos de los documentos de una colección para decidir qué documentos dentro de esta co lec ción es tán más re la cio na dos con una peti ción de bús que da dada. En su lu gar, se constru ye porcada docu men to una re presen tación la cual es la que realmente se emplea al momento de la bús que da. A la ta rea de cons truir las re pre sen taciones de los do cu men tos se les denomina procesodeindexación. Di cho pro- ce so se rea li za una sola vez y con an te rio ri dad a cual quier con sulta.

El in te rés del pre sen te tra baj es so bre los SRI que emplean índices para la búsqueda de la información y, en particular, sobre aquellos que emplean grafmentos de palabras para la crea ción de los ín di ces. ${ }^{(4}$ y 5)

La organización de este artículo es como sigue: Las dos pri me ras sec ciones es tán de dicadas a pre sen tar al gu nos de los con cep tos, definiciones y técnicas que se emplean en los SRI por in de xa cion y que, como se verá, se rán ex tra po la dos para el caso de los SRI por in dexación de triadas. En las siguientes tres secciones se ex po nen las ideas, que aquí se presentan como originales, para una posible op ción en el de sa rro llo de unSRI por in de xación de tria das. En las sec cio nes 6 y 7 se presen tan los al go rit mos de in de xa ción y de búsqueda para el caso de un SRI por indexación 
de tri das. La Sec ción 8 mues tra una prue ba de evaluación de un SRI por indexación de triadas, ba sa do en los al go rit mos de las sec ciones 6 y 7 . En la sec ción 9 se re su men al gu nos trabajos de interés a futuro. Finalmente, en la sec ción 10 se presen tan al gu nas con clu siones sobre el tema.

\section{INDEXACIÓN MANUALY AUTOMÁTICA}

Una de las ma ne ras más co mu nes para ob tener la re presen ta ción de un do cu men to es mediante una lista de tér mi nos o palabras clave, extraídas a partir del documento, que describan lo me jor po sible el con te ni do de di cho docu men to y que ade más con tribu yan a di fe renciaréste del res to de la co lec ción. (figural).

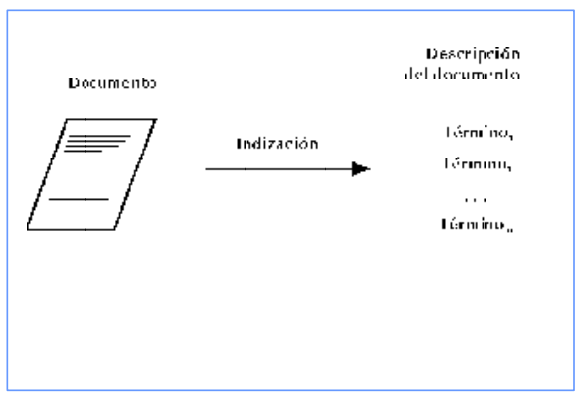

Figura1.

La in de xa ción de un do cu men to como una lis ta de tér mi nos.

Re sul ta eviden te que los tér mi nos que ha brán de ser em plea dos para cons truir las re pre sentaciones, deben ser establecidos con anteriori dad a la cons truc ción de cual quier re pre sentación. Al conjunto de términos que serán em plea dos para cons truir las re pre sen tacioes de los documentos se les denomina lenguaje de in dexación. Es im por tan te pun tua li zar que la elec ción del len gua je de in de xa ción es crut cial para lo grarel buen de sem pe ño de unSRI.

El proce so de in de xa ción pue de ser rea li za do de manera manual o automática. Ambas tienen como fin ex traer del tex to del do cu men to fuen te undocumen tore presen tativoformado por términos del lenguaje de indexación. La principal diferencia que puede hacerse entre estos dos tipos de indexación es que la indexación manual se realiza como su nombre lo indica, en forma manual, teniendo por esto que requerir personal capacitado. Por otro lado, cuan do la asig na ción de iden ti fica do res de contenido se realiza con ayuda de equipo de cóm pu to, la ope ración se lla maindexación automática.

En la indexación manual existe una serie de herramientas que permitenal in dexadorcontro lar el pro ce so de in de xa ción, como son lis tas de terminología, manuales de ins truc ción yprincipalmente hojas de trabajo estructura- das para registrar los productos de indexación,. También suelen utilizarse notas que permitan de fi nirel sig ni fica do y la in ter pretación de cada uno de los términos indexados permitidos en un documento dado.

Para realizar la indexación automática existen va rios al go rit mos, los cua les tie nen como fi nalidad de ter mi nar qué ter mi nos del len gua je de indexación se encuentran presentes en un documento dado. Existen algoritmos de in de xa ción muy sim ples, los cua les se li mi tan a ide xar un do cu men to con un tér mi no dado si este tér mi no (o un frag men to de las pri me ras le tras de éste) apa re ce tal cual en el do cu mento. Exis ten por otro lado, al go rit mos de ide xación muy so fis ti ca dos, los cua les son ca paces de "com pren der" la mor fo lo gía del idio ma en que es tán es cri tos los do cu men tos; por tal razón, es tos al go tir mos son ca pa ces de op ti mizar la lis ta de tér mi nos de la re pre sen ta ción de undocumen to.

\section{LA RECUPERACIÓN DE LOS DOCUMENTOS}

Como ya se mencionó, los SRI no utilizan, generalmente, de manera directa los documen tos para efec tuar las bús que das, en su lugar em plean las re pre sen ta cio nes de los do cur mentos paradecidir qué documentos en la colección son más afines a una petición de bús que da dada. Así pues, es ne ce sa rio que un SRI cuente con una forma de determinar la afinidad de las re presen taciones de los documen tos con los tér mi nos pre sen tes en la pe tición de búsqueda. La manera más común de realizar esta determinación, es simplemente con si de rar como más afi nes a aque llas re presentaciones que contengan el mayor número de tér mi nos pre sen tes en la pe ti ción. Exis ten sin embargo, técnicas para cuantificar la seme jan za en tre una re pre sen ta ción y los tér minos de la pe ti ción de bús que da. En es tas téc nicas no solo se toma en cuen ta que un tér mi no esté o no esté pre sen te en una re pre sen ta ción, sino que además se le asigna a cada término un determinado peso por medio del cual se podera la importancia de cada término en la in de xa ción del do cu men to. El cri te rio que común men te se em plea para pon de rar un tér mino es a tra vés de su fre cuen cia de ocu rren cia a lo lar go de la co lec ción de do cu men tos y dentrode cada do cu men to enpar ti cu lar; don de se considera que un término tiene mayor peso para la representación del documento cuanto más frecuente sea éste dentro del mis mo documento y menos lo sea dentro de la colección. ${ }^{(7)}$ Usando un esquema de ponderación de términos, la representación de un documento pudiera estar dada por un vector de ponderaciones de tér mi nos, comose mues tra en la siguiente figura:

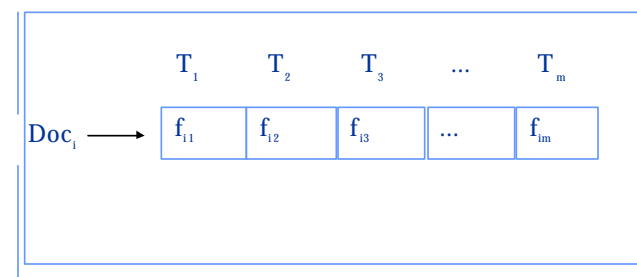

Figura 2.

Cons truc ción de la re pre sen ta ción del i-ési mo do cu men to de una co lec ción, don dem es la car dinalidad del len gua je de in de xa ción y $f_{i j}$ es la pon de ra ción del Tér mi no $T_{j}$ den tro del i-ési mo docu men to $d o c_{i}$.

Bajo un esquema de ponderación de términos, la cuan ti ficación de la se me jan za de la repreentación de un documento y los términos pre sen tes en la pe ti ción de bús que da, se rea liza a través de una medida de similitud, la cual no es otra cosa que una expresión algebráica en donde, de alguna manera, se hacen intervenir las ponderaciones de los términos de la re pre sen ta ción y los tér mi nos de la pe tición para evaluar nu mérica men te su se me janza (figura 3). De esta manera, mediante esta medida de similitud de SRI son más "cercanos" a una petición de búsqueda. Aún más, usando este criterio de cercanía se puede construir una lista de do cu men tos que se encuentre ordenada en forma decreciente con respecto a sus valores de semejanza.

\begin{tabular}{|c|c|c|c|c|c|c|}
\hline \multirow{3}{*}{$\begin{array}{l}\mathrm{Doc}_{1} \\
\mathrm{Doc}_{2}\end{array}$} & $\mathrm{~T}_{1}$ & $\mathrm{~T}_{2}$ & $\mathrm{~T}_{3}$ & $\ldots$ & $\mathrm{T}_{\mathrm{m}}$ & $\begin{array}{l}\text { Valores de } \\
\text { Similitud }\end{array}$ \\
\hline & $f_{11}$ & $f_{12}$ & $\mathrm{f}_{13}$ & $\ldots$ & $\mathrm{f}_{\mathrm{tm}}$ & $\mathrm{VS}_{1}$ \\
\hline & $f_{21}$ & $\mathrm{f}_{22}$ & $\mathrm{f}_{23}$ & $\ldots$ & $\mathrm{f}_{2 \mathrm{~m}}$ & $\mathrm{VS}_{2}$ \\
\hline & & & $\vdots$ & & & $\vdots$ \\
\hline $\operatorname{Doc}_{n}$ & $f_{n 1}$ & $\mathrm{f}_{\mathrm{n} 2}$ & $\mathrm{f}_{\mathrm{n} 3}$ & $\ldots$ & $\mathrm{f}_{\mathrm{nm}}$ & $\mathrm{VS}_{\mathrm{n}}$ \\
\hline Pet & $\mathrm{p} 1$ & p2 & p3 & $\ldots$ & $\mathrm{p}$ & \\
\hline
\end{tabular}

Figura 3.

Cál cu lo de los va lo res de si mi li tud, don de $p j$ es la pon de ra ción del tér mi no $T j$ den tro de la pe ti ción $P e t$ y $V S i$ es el va lor de si mi li tud en tre el do cu men to Doci y la pe ti ción Pet.

Exis ten diferentes ma ne ras de de fi nir una medidadesmilitud. (7) Una de uso muy fre cuen te es la medida coseno, en donde el valor de similitud $\mathbf{V S} \mathbf{i}$, para cada representación de documento Doci y la representación de la petición Pet, se calcula como el coseno del ángu loque for man am bas re presen ta ciones:

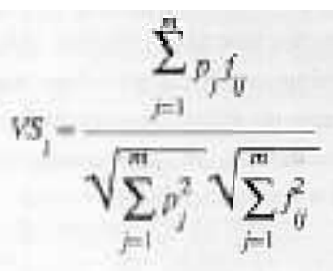


Usan do una me di da de si mi li tud comola de fini da an te rior men te, se pue de es ta ble cer un lí mite para el conjunto de documentosrecuperados. Por ejemplo, se podría establecer que el conjun to de docu men tos recu pe ra dos sean aquellos cuyas representaciones mostraron una valor de similitud mayor a 0.7 , con respec to a la re pre sen ta ción de la pe ti ción. Otra al ternativa, podríaserpresentarsolamentelos $N$ primeros documen toscu yas re presentaciones mostraron mayor valor de similitud con respecto a la representaciónde la petición.

Los docu men tos recu pe ra dos pue den serconvenientemente presentados al usuario en orden de cre cien te con res pec to a los va lo res de similitudque mos tra ron susre presen taciones con la pe ti ción de bús que da. Esto es de im portancia especial en una situación de re cuperación interactiva, pues nuevas y mejores formulaciones de peticiones de búsqueda pue den ser cons trui das a par tir de la in for mación ob tre nida de los documen tos previamenterecuperados(retroalimentación).

\section{IN DE X A CIÓN POR TRIA DAS}

La in de xa ción por tria das es una téc ni ca de inde xa ción au to má ti ca, la cual con sis te en descomponer cada una de las palabras no nulas (es decir palabras diferentes a los artículos, pre posi ciones, con jun ciones, etc., de un idioma) de un documento en triadas de letras y uti li zar a la fre cuen cia de ocu rren cia de és tas para cons truir un pa trópn de tria das (his to grama) que conformará la indexación del documen to. Para en ten der con ma yor pre ci sión lo anterior se convendrá que una triada válida para una pa la bra dada es cual quier grag men to continuo de tres letras que esté contenido en esta palabra. Por ejem plo el con jun to de triadas que pue de ser cons trui do en base a las palabras sofia unam ( $\sin$ acento) es:

\section{sof, ofi, fia, una y nam}

Esta técnica cuenta con dos características que la hacen muy atractiva. La primera es la facilidad de su imple men ta ción y la se gun day más importante es que el proceso de indexación deja de depender casi en su totalidad de la lengua alfabética en que estén escritos los do cu men tos. La ra zón por la que el proce so de in de xa ción por tria das si gue de pen dien do del idioma es debido a que no todas las posibles combinaciones de tres letras (triadas) son igualmente válidas en todas las lenguas alfabéticas.

Naturalmente se podría salvar esta dependencia si se permitiera dar validez a cualquier com bi na ción de tres le tras en cual quier idioma. sin embargo, el número de triadas válidas sería demasidado grande y esto implicaría que los archivos que se generaran como resultado del proceso de indexación

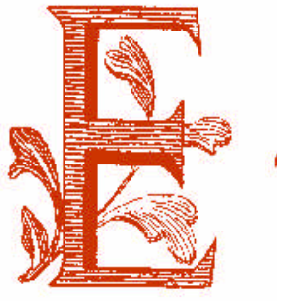

\section{“ La indización por tríadas es una técnica automática, la cual} consiste en descomponer cada una de las palabras no nulas de un documento en tríadas de letras y utilizar la frecuencia de ocurrencia de éstas para construir un patrón de tríadas que conformará la indización del documento"

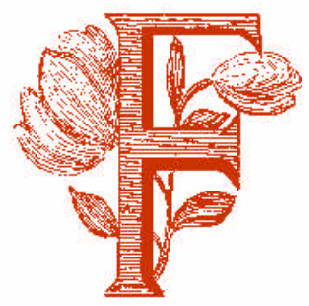

se rían tam bién gran des. Por tal ra zón, es recomendable realizar un análisis estadístico de las triadas válidas del idioma alfabético en que está es cri ta la co lec ción de do cu mentos. Este análisis no sólo permitirá determinar las tria das vá li das sino tam bién co no cer con qué frecuencia se usa una triada en el idio ma. Esto úl ti mo es de gran uti li dad, pues en este trabajo se ha su pues to que el con jun to de triadas válidas (lenguaje de indexación portria das) con que ha brán de ser in de xa dos to dos los do cu men tos, no de ben in cluir triadas muy poco fre cuen tes ni las muy fre cuentes. Tres son las razones por las que se cree que deben ser eliminadas estas triadas del len gua je de in de xa ción. La pri me ra ra zón es porque se piensa que las triadas son muy poco frecuentes, por su misma rareza, pueden ser eliminadas sin mayor perjuicio al rendimiento de un SRI por indexación de tria das. La se gun da ra zón es por que se piensa también, que las triadas muy frecuentes aparecerán con toda seguridad en todos los documentos de la colección, lo cual hace a estas triadas ma los can dida tos paracaracterizar y diferenciar los documentos de la colección. La tercera razón es una consecuencia de las dos anteriores, pues al eliminar aque llas tria das poco fre cuen tes así como las muy frecuentes, el conjunto de triadas válidas (fil tra das), que con for ma rán el len gua je de indexación, puede reducirse considerablemente.

El aná li sis es ta dís ti co de las tria das en el caso del idioma español, fue realizado con ayuda de un conjunto de artículos de periódicos cuyo volumen fue de aproximadamente 8 Mbytes (un mes de artículos). Este archivo muestra se pre proce só an tes de su análi sis de tal ma ne ra que se eli mi nó todo acen to y sus tituida toda ñ por $n$, pues se considera que los acentos así como las ñ's dentro de los documen tos es cri tos con tri bu yen muy poco a la interpretación del mismo. como resultado del aná li sis es ta dís ti co se en con tró que em pleando este ar chi vo mues tra exis tían 6262 tria das vá li das en este es pa ñol sin acen tos, cu yas frecuencias de aparición oscilaban de 1 hasta 41226. Para re du cir el con jun to de tria das válidas se decidió introducir un filtro para las 6262 tria das, de tal ma ne ra que fue ron con sideradas como válidas sólo aquellas triadas cuyas frecuencias se encontraran entre 7 y 3000. Es importante acla rar, que las ca rac terísticas de este filtro han sido el resultado de estimaciones al observar el reporte estadístico de las triadas y de evaluaciones de rendimiento de un SRI por indexación de triadas. Bajo la condición de este filtro, seestable ció finalmente un lenguaje de indexación por tria das con 3797 tria das vá li das las cua les han sido la base para la cons truc ción de las re presentaciones de los documentos. 


\section{CONS TRUC CIÓN DE LAS REPRESENTACIONES DE LOS DOCUMENTOS}

Una vez que ha sido establecido el conjunto de tria das que con for ma rán el len gua je de indexación es posible construir representaciones o indexaciones de los documentos basados en este lenguaje de indexación. Ahora bien, de bi do a que en este caso el len gua je de in de xa ción no está con for ma do por tér mi nos o pa la bras, en ton ces la cons truc ción de las representaciones no deberá ser una extrapolación di rec ta de la téc ni ca des cri ta en lafigura 2. Así pues, habrán de efectuarse algunos cambios y ajustes. El primer cambio es que ahora, cada representación estará conformada por uno o más vec to res (vec to res de pon dera ción de tria das), en don de el nú me ro de vectores dependerá de la longitud promedio de los documentos en la colección. Lo anterior se debe a que no es con ve nien te usar un solo vector para la representación de documentos muy extensos, pues, en este caso, se corre el ries go de que la re pre sen ta ción re por te la presencia de la mayoría de las triadas válidas. Consecuentemente se estaría consideando casi a cual quier pa la bra o tér mi no como presente dentro del documento. Una manera de solucionar este problema es segmentar los documentos muy extensos y construir para cada uno de estos segmentos un vector de pon de ra ción de tria das. Una for ma de rea li zar la seg men ta ción po dría ser por ejem plo uti lizar primero solamente las primeras $N$ palabras no nu las del do cu men to para la cons trucción del primer vector de ponderación de triadas. Después se continuaría con las siguientes $N$ pa la bras no nu las para cons truir el segundo vector. Esto se repetiría sucesivamente hasta la construcción del r-ésimo vector, don der se ría el nú me ro de seg men tos que se emplearían por documento. Lo an teriorse esquematiza en la siguiente figura:

\begin{tabular}{|c|c|c|c|c|c|}
\hline $\mathrm{T}_{1}$ & $\mathrm{~T}_{2}$ & $\mathrm{~T}_{3}$ & $\ldots$ & $\mathrm{T}_{\mathrm{m}}$ & \\
\hline $\mathrm{f}^{1}{ }_{\mathrm{i}}$ & $\mathrm{f}_{\mathrm{i} 2}^{1}$ & $\mathrm{f}_{\mathrm{i} 3}^{1}$ & $\cdots$ & $\mathrm{f}_{\mathrm{im}}^{1}$ & Segmento 1 \\
\hline $\mathrm{f}^{2} \mathrm{i}$ & $\mathrm{f}^{2} \mathrm{i}$ & $\mathrm{f}^{2} \mathrm{i}$ & $\ldots$ & $\mathrm{f}^{2}{ }_{\text {im }}$ & Seg men to 2 \\
\hline
\end{tabular}

Doc $_{\mathrm{i}} \rightarrow$

\begin{tabular}{|l|l|l|l|l|}
\hline $\mathrm{f}^{\mathrm{r}} \mathrm{i}$ & $\mathrm{f}^{\mathrm{r}} \mathrm{i}$ & $\mathrm{f}^{\mathrm{r}} \mathrm{i} 3$ & $\cdots$ & $\mathrm{f}^{\mathrm{r}} \mathrm{i}$ \\
\hline
\end{tabular}

\section{figura 4}

Cons truc ción de la re presnta ción deli-és imo do cut men to de una co lec ción usan do in di za ción por tríadas, don de $\mathbf{m}$ es la car di na li dad del len gua je de indi za ción por tría das y $\mathbf{f}_{\mathbf{i j}}$ es la pon de ra ción de la tríada $\mathbf{T}_{\mathbf{j}}$ den tro del $\mathbf{k}$-és imo seg men to del $\mathbf{i}$-ésimo documento doc
Aho ra bien, la pre gun ta que sur ge es ¿c uñal es el núme roideal $N$ de pa la bras no nu las que se deberán con si de rar para cada seg men to? Dar una respuesta a esta pregunta probablemente no sea algo sencillo. Sin embargo, una estima ción fue he cha como si gue: Se le ye ron, en orden de aparición, las palabras no nulas de un tex to cual quie ra. Cada vez que se leyó una de es tas pa la bras, se ex tra je ron sus tria das vá li das, de las cua les se lle vó re gis tro con jun tamen te con las tria das vá li das de pa la bras leídas con an te rio ri dad. La lec tu ra de pa la bras se con ti nuó has ta que se en con tró una pa la bra en la cual al me nos dos de sus tria das vá li das, ya habían sido registradas por otra u otras palabras anteriores. Este experimento se repitió para diferentestextos en es pañol y se en contró que aproximadamente 170 era el número prome dio de pa la bras no nu las que eran leídas an tes de term nar. Si con si de ra mos que aproxi mada men te ocu rren 5 pa la bras no nu las en un ren glón de tex to nor mal, en ton ces 170 pala bras nonu las equi va len aproxi ma da men te a $3 / 4$ de una cuartilla de tex to nor mal.

Otro cambio en cuanto a la construcción de las re presen taciones de los docu men tos, se refie re al es que ma de pon de ra ción para las triadas. En este caso se de ci dió usar sim ple men te como ponderación a la frecuencia de cada tria da den tro de cada seg men to. Sin em bargo, debido a que sosn relativamente pocas las tria das que se rán con ta bi li za das por seg mento, se decidió acotar los valores $f^{k} i j$ como sigue:

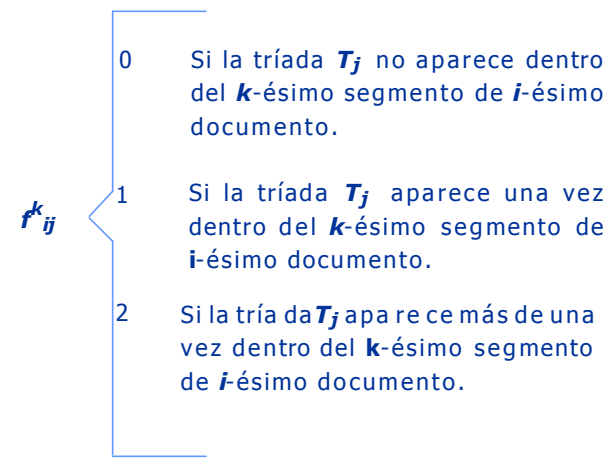

Debe observarse que al ser limitados así los va lo res de $f^{k}{ }_{i j}$, en ton ces se rán su fi cien tes dos bits para codificar cualquiera de sus valores. Esto úl ti mo es de gran im por tan cia si se de sea optimizar el tamaño de las representaciones de los documentos.

Una vez resuelto el pro ble ma de la cons trucción de las re pre sen ta cio nes de los do cu mentos, es posible conceptualizar el conjunto de documentos indexados como una matriz, la cual llamaremos matriz de indexación. Esta matriz será construida simplemente como la concatenación sucesivade las re presen taciones de los do cu men tos en la co lec ción, esto se esquematizaen la siguientefigura:

\begin{tabular}{|c|c|c|c|c|}
\hline $\mathrm{T}$ & $\mathrm{T}_{2}$ & $\mathrm{~T}_{3}$ & $\ldots$ & $\mathrm{T}_{\mathrm{m}}$ \\
\hline $\mathrm{f}_{11}^{*}$ & $f_{12}^{*}$ & $\mathrm{f}_{13}^{*}$ & $\ldots$ & $\mathrm{f}_{1 \mathrm{~m}}^{*}$ \\
\hline $\mathrm{f}_{21}^{*}$ & $\mathrm{f}_{22}^{*}$ & $\mathrm{f}_{23}^{3}$ & $\ldots$ & $\mathrm{f}_{2 \mathrm{~m}}^{*}$ \\
\hline$f_{(n, p) \mid 1}^{*}$ & $f^{*}{ }_{(\mathrm{n} g r) / 2}$ & $\mathrm{f}_{\left(\mathrm{n}, v_{\mathrm{r} / 3}\right.}^{*}$ & $\ldots$ & $\mathrm{f}_{\left(\mathrm{n}, \rho_{\mathrm{f} r) \mathrm{m}}\right.}^{*}$ \\
\hline
\end{tabular}

\section{figura 5}

Cons truc ción de la ma triz de in di za ción como la con ca te na ción su ce si va de to das las re pre sen taciones de los do cu men tos de una co lec ción de $n$ documen tos, don de $f^{*}{ }_{s}=f^{k}{ }_{i j}$ para $s=(i-1) \cdot r+k$ con $1 \leq i \leq$ $n, 1 \leq j \leq m, 1 \leq k \leq r$ y $1 \leq s \leq n \cdot r$

Debido a que generalmente una petición de búsqueda estará conformada por tan sólo algunas palabras no nulas, entonces la construc ción de la re pre sen ta ción de una pe ti ción se hará de manera análoga a la construcción de la representación de un documento, sólo que aho ra se em plea rá un sólo seg men to para con ta bi li zar las tria das que se for men a par tir de las palabras de la petición(figura 6).

\begin{tabular}{|l|l|l|l|l|}
\hline \multicolumn{1}{|c}{} & $\mathrm{T}_{1}$ & \multicolumn{1}{c|}{$\mathrm{T}_{2}$} & \multicolumn{1}{c|}{$\mathrm{T}_{3}$} & \multicolumn{1}{c|}{$\mathrm{T}_{\mathrm{m}}$} \\
\hline Petición $\longrightarrow \mathrm{p}_{1}$ & $\mathrm{p}_{2}$ & $\mathrm{p}_{3}$ & $\ldots$ & $\mathrm{p}_{\mathrm{m}}$ \\
\hline
\end{tabular}

\section{figura 6}

Cons truc ción de la re pre sen ta ción de la pe ti ción de bús que da, don de las pon de ra cio nes $p_{j}$ se obtienen de ma ne ra aná lo ga a las pon de ra cio nes $f^{k}{ }_{i j}$.

Una con si de ra ción fi nal acer ca de la ma triz de in de xa ción es acer ca de su po ro si dad, ya que en ge ne ral la probabilidad de no pre sen cia de una determinada triada en un determinado seg men to de un do cu men to será ma yor que la probabilidad de pre sen cia. Así pues, es re comen da ble para evi tar que la ma triz de in de xa ción sea muy gran de, uti li zar có di gos de compresión tales como los códigos de Hoffman ${ }^{(8)}$ para la codificación de esta matriz de indexación. Es cla ro que al com pri mir la ma triz de in de xa ción se afec ta rán an tes de iden ti ficar a los do cu men tos cu yas re presenta ciones mues tran los va lo res más gran des de similitud con res pec to a la re presen ta ción de la búsqueda. Una manera de lograr que los tiem pos de bús que da no se vean tan afec ta dos con esta medida es no comprimir a la matriz de indexación como un todo y, en su lugar, rea li zar la com pre sión de la ma triz por columnas independientes. De esta forma, cada vez que sea ini cia da una bús que da será ne ce sa rio sólo descomprimir, de la matriz de indexación, aque llas co lum nas cu yas tria das correspon dien tes se en cuen tran pre sen tes en la pe tición de búsqueda. Esto último será expuesto con ma yor de ta lle en las sec cio nes 6 y 7 . 


\section{ESPECIFICACIÓN DE LA MEDIDA DE SIMILITUD}

Da das lasex plicaciones y de fi ni ciones dadas en la sección anterior, la pregunta que surge naturalmente es ¿de qué manera se decidirá cuándo un determinado documento en la colección es más si mi lar a una pe ti ción de búsque da dada? Tal pre gun ta que da rá re suel ta al definir una medida de similitudquecuan tifique la si mi li tud en tre la re pre sen ta ción de un do cu men to y la re pre sen ta ción de la pe ti ción. Una opción para la medida de similitud, podría ser la medida coseno descrita anteriormente. Sin embargo, esta medida empleada en nues tro caso, to ma ría en cuen ta las tria das no pre sen tes en la pe ti ción para cuan ti fi car la similitud. ${ }^{(9)}$ Así pues, sería deseable contar con una me di da de si mi li tud que, sin de jar de considerar a la frecuencia de las triadas, le die ra másim por tan cia a los seg men tos del docu men to que con ten gan el ma yor nú me ro de tria das di fe ren tes que es tén pre sen tes en la petición. Ahora bien, como los valores de las ponderaciones de las triadas $f_{i j} q$ quedaron limitados a 0,1 ó 2 , entonces una opción para cuan ti ficar la similituden tre un se gu men to de documento y una petición de búsqueda $\left(\boldsymbol{V} \boldsymbol{S}^{*}{ }_{\mathbf{s}}\right)$ podría ser a través de la siguiente expresión:

$$
v_{s} \cdot=\sum_{j=1}^{n} p_{j}, f_{j}{ }^{*}+5 \sum_{j=1}^{*} p_{j}, p_{j} \cdot
$$

Donde $\boldsymbol{P}_{j}$ es igual a cero si $\boldsymbol{p}_{\boldsymbol{j}}=\boldsymbol{0}$, e igual a uno si $\boldsymbol{p}_{\boldsymbol{j}}$ es diferente de cero. De maneraanáloga se de fi ne $\boldsymbol{F}^{*}{ }_{s j}$. A tra vés de la ex pre sión an terior es posible determinar qué segmentos de la ma triz de in de xa ción son más se me jan tes a una pe ti ción de bús que da dada. Sin em bargo, lo que real men te in te re saes poder cuan ti ficar la si mi li tud de un do cu men to con una pe ti ción dada $\left(\boldsymbol{V} \boldsymbol{S}_{\boldsymbol{i}}\right)$. Una manera de establecer tal cuantificación podría ser simplemente a través del má xi mo de los va lo res de si mi li tud de los segmentos de un documento (VS ${ }^{*}$ s), lo cual se realiza con la siguienteexpresión:

$$
w_{i}=\operatorname{Mar}\left(\sum_{j=1}^{n} p_{j} \cdot f_{p}^{k}+5 \sum_{k=1}^{N} P_{j} \cdot F_{k}{ }^{k}\right)_{k=1}
$$

Donde nuevamente $\boldsymbol{F}^{\boldsymbol{k}} \boldsymbol{i j}$ se define análogamente a $\boldsymbol{P}$.

\section{Algoritio DE INDEXACIÓN}

El proceso para generar la matriz de indexación es sumamente complejo y su complejidad radica principalmente en la necesidad, como ya se hizo mención, de comprimir por se pa ra do las co lum nas de la ma triz. Para lle- var un con trol pre ci so del proce so de in de xación se empleó una estructura de datos cuya función es semejante a la que realiza la Process Status Word (PSW) en el caso del fun cionamiento global, a ni vel de hard ware, de una computadoradigital. Estaes truc tu rade da tos, la cual fue denominadacon el nombre de estado, tie ne con cre ta men te como fun ción la de re gis trarel es ta do fi nal de la ma triz de in de xación después de la última indexación. Asi pues la in for ma ción que esta es truc tu ra estado maneja es la siguiente:

- Un contador global, con el cual se lleva cuenta del número total de segmentos de do cu men to que han sido in de xa dos has ta el momento.

- -Un apun ta dor por cada co lum na de la matriz de in de xa ción. A travñes de es tos apuntadores se especifica el inicio de cada colum na den tro de la ma triz de in de xación. Se debe re cor dar que las co lum nas de la matriz de in de xa ción se ha yan com pri mi das y por tal razón no existe una regularidad en cuanto al tamaño y ubicación de cada colum na den tro del ar chi vo que con ten drá a la matriz.

- Un con ta dor por cada co lum na de la ma triz de indexación, este contador registra el tamaño en bytes de la columna comprimida que le corresponda. La función que estos contadores rea lizan es muy im portan te pues a través de éstos puede saberse con precisión el número de bytes que deben ser leídos de la matriz de indexación cuando se desee manipular una de sus columnas.

El proceso de indexación de la colección de docu men tos es en esen cia un proce so se cuencial en el sentido de que la indexación de la co lec ción se rea li za al in de xar uno por uno todos los segmentos de todos los documentos de la co lec ción. De esta ma ne ra la in de xa ción de la co lec ción es el re sul ta do de una suce sión de in de xa ciones. en cada una de es tas in de xaciones se indexa uno y sólo uno de los segmentos de un documento dado de la coleccióny el resultado de esta indexación se agrega como un nuevo renglón al final de la matriz de in de xa ción. Los pa sos que se realizan para in de xary agre gar un nue vo seg mento al conjunto de segmentos ya indexados, son los siguientes:

Se eli mi nan del tex to del seg men to a in dexar las palabras nulas.

par tir del tex to re sul tan te del paso an te rior, se construye un vector de frecuencias de triadas, el cual es equivalente a cualquier ren glón de la ma triz de in de xa ción de la $f i$ gura 5.

- Con la ayu da de la es truc tu raestado se reali zan para cad j-és ima co lum na de la ma triz de indexación de los siguientes 4 pasos:
Se lee laj-ési ma co lum na de la ma triz de index qa ción la cual es de com pri mi da a con tinuación.

A la columna descomprimida se le agrega al final de la $j$-ésima entrada del vector de frecuencias.

Esta columna agrandada es comprimida a continuación para obtener de esta forma una nue va colum na com pri mi da que in clya ya la $j$-ési ma en tra da del vec tor de fre cuencias.

La nue va colum na com pri mi da es aho ra escri ta como par te de una nue va ma triz de indexación.

Una vez que han sido leídas todas las columnas de la matriz de indexación y que también hayan sido escritas todas las colum nas de la nue va ma triz de in de xa ción, se bo rra la ma triz de in de xa ción an te rior y declarada a la nueva matriz como la actual.

La estructura estado es modificada de tal manera que refleje las condiciones de la nue va ma triz de in de xa ción.

\section{Algoritmo de BúSQUEDA}

Una vez que ha sido indexada toda la colección de documentos, es posible la búsqueda de docu men tos. Esta bús que da será rea li zada sobre la matriz de indexación y bajo el siguiente algoritmo:

Se eli mi nan del tex to de la pe ti ción to das las palabras nulas y con el texto restante se construye el vector de frecuencias de triadas de la petición el cual se denotará por $\boldsymbol{P e t}=\left(\boldsymbol{p}_{1}, \boldsymbol{p}_{2}, \ldots, \boldsymbol{p}_{\boldsymbol{m}}\right)$ (donde $\boldsymbol{m}$ es igual a la cardinalidaddel len gua je de in de xación).

Es inicializado con ceros un vec tor el cual deberá tener tantas entradas como renglones en la ma triz. En las en tra das de este vector habrán de depositarse los resultados de las me di das de si mi li tud de cada seg men to de documento con respecto a la petición. Por esta ra zón, este vec tor será de no mi na do como

$$
\left.V S_{1}^{*}, V S_{2}^{*}, \ldots, V S_{r ; n}^{*}\right) \text {. }
$$

Donde $\boldsymbol{n}$ es igual al nú me ro de do cu men tos en la co lec ción y $\boldsymbol{r}$ es el nú me ro de seg mentos por documento.

Con la ayu da de la es truc tu raestado se reali za para cadaj-és ima en tra da del vec tor de frecuencias que sea diferente de cero, los siguientes 2 pasos:

Se lee la $j$-ési ma co lu ma de la ma triz de indexación y se descomprime inmediatamente. Esta columna será denotada por

$$
T_{j}=\left(f *_{i j}, f^{*}{ }_{2 j}, \ldots, f^{*}(r . n) j\right) .
$$




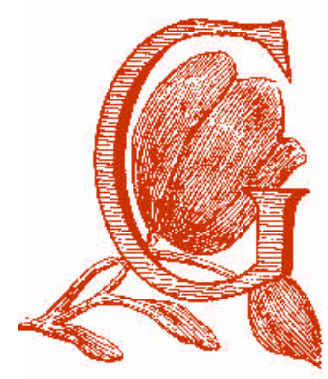

"La indización por tríadas cuenta con dos características que la hacen muy atractiva. La primera es la facilidad de su aplicación y la segunda, y más importante, es que el proceso de indización deja de depender casi en su totalidad de la lengua alfabética en que están escritos los documentos"

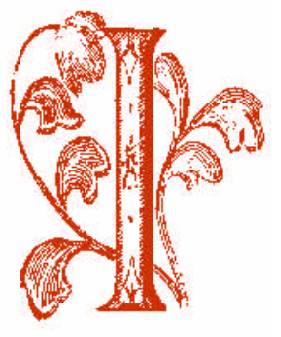

- El vector de similitudes VS ${ }^{*}$ es actualizadocomo sigue:

En donde como antes $F^{*}{ }_{s j=0}$ si $f^{*}{ }_{s j=0}$ y $\boldsymbol{F}^{*}{ }_{s j}=1$ si ${ }^{*}{ }_{s j} 0$.

Se ob tie nen los va lo res de si mi li tud por documento ( $\boldsymbol{V} \boldsymbol{S}_{1}$ ), es decir se obtiene el segmento de valor máximo por cada documento.

$V S_{i}=\operatorname{Max}\left(V S_{(i-1),+1}^{+} V S_{(6-1),-2, \ldots+1}^{+} V S_{i, r}^{+}\right)$
$i=1,2 \ldots, \ldots$

Se ordenan de mayor a menor los valores anteriores y se imprimen finalmente los nom bres de los prime ros $N$ documen tosque correspondan a estos valores ordenados.

\section{EVALUA CIÓNDEL SRI POR IN DE X A CIÓN DE TRIA DAS}

Para la evaluación de un SRI basado en triadas, se empleó un banco de información de más de me dio mi llón de fi chas bi blio grá fi cas del catálogo de la biblioteca de la DGB de la UNAM. Es tas fi chas re gis tra ron una lon gi tud pro me dio de 237 bytes, es de cir, aproxi ma damen te 40 pa la bras nu las o no nu las por fi cha. Este ban co de in for ma ción se in de xó uti li zando la téc ni ca de in de xa ción por tria das des critaanteriormente y de bi do a que en este caso, la lon gi tud pro me dio por do cu men to fue menor de 170 pa la bras no nu las, se em pleó en la indexación de cada ficha un solo segmento (es de cir $r=1$ ). Como re sul ta do se ob tu vo una matriz de indexación cuyo tamaño fue de apro xi ma da men te 45 Mbytes. Dado que el tama ño del ban co de ien for ma ción es de aproximadamente 122 Mbytes esto significa que la ma triz de in de xación ocu pa aproxi ma da mente el equivalente al $38 \%$ de este espacio. La supercomputadora Cray $Y-M P / 432$ y el tiempo de CPU que requirió para indexar todo el banco fue de aproximadamente 7 horas. Podría parecer que es demasidado el tiempo de indexación y sobre todo para un equipo de còm pu to como lo es una Cray, sin em bar go, a este res pec to se de ben men cio nar tres pun tos. pri me ro, la in de xa ción del ban co de in for mación se realiza sólo una vez. Segundo, la razón por la que se em pleó la Cray obe de ce más a una ne ce si dad de con tar con una bue na cantidad de dis cos y no a una ne ce si dad de ve locidad de proceso. Debido a la naturaleza del algoritmo de indexación se estima que el tiempo de indexación en otros equipos de cómputo no será extremadamente diferentes al reportado para la Cray. Tercero, por la men ra en que se ha he cho la im ple men ta ción del algoritmo de indexación, los resultados que con este alforitmo se obtienen son independientes de la plataforma de cómputo que se em plee para ge ne rar los y por esta ra zón, es- tos resultados pueden, sin ningún problema, ser usados para la búsqueda de documentos bajo otra plataforma de cómputo incluyendo computadoraspersonales.

Por otro lado, es importante mencionar que den tro de este ban co de in for ma ción exis ten fichas bi bli grá fi cas es cri tas en otros idio mas dife ren tes al es pa ñol como son el in glés, fran cés e ita lia no. A pe sar de te ner co no ci mien to de lo an te rior, se em pleó para la in de xa ción del banco de información el lenguaje de indexación cons trui do es pe cial men te para el idio maes pa ñol. Dos son las ra zo nes por las que se de ci dió indexar al ban co de in for ma ción con este lenguaje de indexación, la primera razón es simple men te por que la gran ma yo ría de las fi chas bibliográficas están escritas en es pa ñol, la segun da ra zón es por que se pen só que se ría in teresante evaluar el comportamiento del SRI cuan do al gu nos de los do cu men tos de la co lección no estuvieran escritos en español.

Para lle var a cabo la eva lua ción fue ne ce sa rio primeroconfor mar un pa drón he te rogéneode pe ti cio nes de bús que da y em plear este pa drín en las pruebas de evaluación. Este padrón de peticiones fue conformado con ayuda de un programa de computadora que "analizó" la colección de do cu men tos (más de me dio millón de fi chas) y en base a este análi sis se es ta bleció este padrón. La manera en que operó este programa sobre la colección de documen tos fue como si gue:

1. Se eli gie ron alea to ria men te 1245 documentos de la colección.

2. Para cada uno de los 1245 documentos se lec cio na dos en el paso an te rior, se cons tru yó una pe ti ción de bús queda la cual fue construida como una ca de na de en tre una y cua tro pa la bras que fueron extraídas al azar de cada documento.

A continuación se muestra un fragmento del padrón de peticiones que fue construido con ayuda de este programa de computadora (el número al principio de cada petición indica cuan tas pa la bras con for man a la pe ti ción).

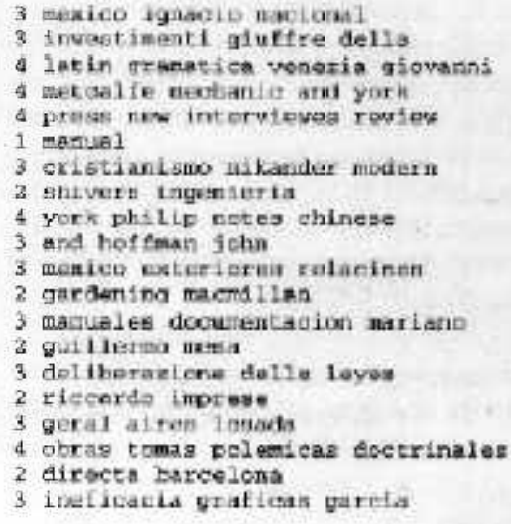


De bido a la im posibilidad de rea li zar manuat men te 1245 pe ti cio nes de bús que da y eva luar los re sul ta dos, se de sa rrolló otro pro gra ma de cóm pu to que rea li za ra este tra ba jo de eva luación. Este nue vo pro gra ma ana li za, para cada petición de búsqueda en elpadrón, entregada por el SRI. Este análi sis con sis tió en de ter minar el número que ocupaba aquel primer documento, dentro de la listra entregada por el SRI, que contiviera todas las palabras incluídas en la pe ti ción de bús que da. Se de ci dió por simplicidad que el programa sólo analizara los diez prime ros docu men tos de la lis ta or dena da, de tal ma ne ra que de no en con trar se un documento que contuviera todas las palabras de la pe ti ción se re por ta ría como una bús queda falli da.

A continuación se muestra un fragmento del reporte generado por este programa evaluador

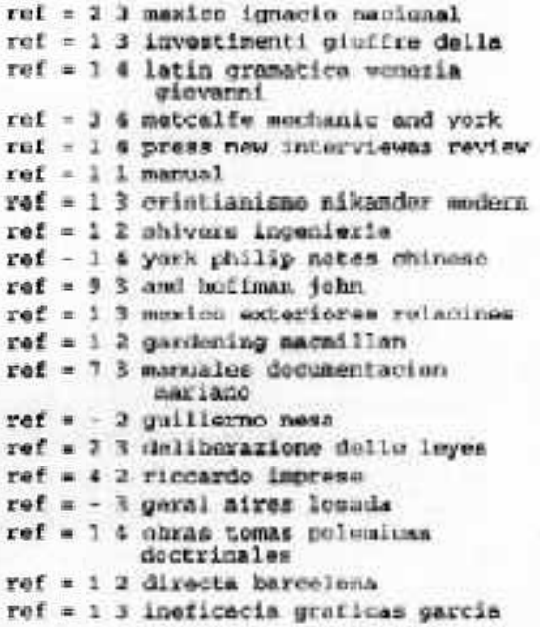

Como puede observarse este fragmento es casi igual al frag men to del pa drón de pe ti ciones salvo por la primera columna numérica. En esta co lum na se in di ca, para cada pe ti ción, el número que ocupa el primer documento, dentro de la liesta ordenada entregada por el SRI, que con tie ne to das las pa la bras usa das en la bús que da. Cuan do un guión apa re ce en lugar de un número, en estacolum na, sig nifica que no se en con tró tal do cu men to den tro de la lista de los diez primeros documentos.

Una manera de resumirlos resulta dos con tenidos en el reporte de evaluación es a través de las siguientes tablas:

\begin{tabular}{|c|c|c|c|}
\hline \multicolumn{4}{|c|}{304 prue bes do 1 pelobra } \\
\hline 1 & 1 & 185 & $60.88 \%$ \\
\hline 1 & 2 & 34 & $11.18 \%$ \\
\hline 1 & 3 & 11 & $3.62 \%$ \\
\hline 1 & 4 & 7 & $2.30 \%$ \\
\hline 1 & 5 & 4 & $1.32 \%$ \\
\hline 1 & 6 & 2 & $0.66 \%$ \\
\hline 1 & 7 & 3 & $0.89 \%$ \\
\hline 1 & E & 6 & $1.97 \%$ \\
\hline 1 & 8 & 1 & $0.33 \%$ \\
\hline 1 & 10 & 5 & $1.84 \%$ \\
\hline 1 & - & 48 & $15.13 \%$ \\
\hline
\end{tabular}

\begin{tabular}{|l|l|l|l|}
\hline \multicolumn{5}{|c|}{304 pruebas do 2 palabraz } \\
\hline 2 & 1 & 176 & $57.89 \%$ \\
\hline 2 & 2 & 41 & $13.49 \%$ \\
\hline 2 & 3 & 16 & $5.26 \%$ \\
\hline 2 & 4 & 9 & $2.96 \%$ \\
\hline 2 & 5 & 5 & $1.64 \%$ \\
\hline 2 & 8 & 4 & $1.32 \%$ \\
\hline 2 & 7 & 8 & $2.63 \%$ \\
\hline 2 & 8 & 4 & $1.32 \%$ \\
\hline 2 & 9 & 4 & $1.32 \%$ \\
\hline 2 & 10 & 2 & $0.66 \%$ \\
\hline 2 & - & 35 & $11.61 \%$ \\
\hline
\end{tabular}

\begin{tabular}{|l|l|l|l|}
\hline \multicolumn{5}{|c|}{325 pruebas da 3 palabras } \\
\hline 3 & 1 & 249 & $76.62 \%$ \\
\hline 3 & 2 & 27 & $8.31 \%$ \\
\hline 3 & 3 & 11 & $3.38 \%$ \\
\hline 3 & 4 & 3 & $0.92 \%$ \\
\hline 3 & 6 & 7 & $2.5 \%$ \\
\hline 3 & $E$ & 3 & $0.92 \%$ \\
\hline 3 & 7 & 4 & $1.23 \%$ \\
\hline 3 & 6 & 3 & $0.92 \%$ \\
\hline 3 & 8 & 6 & $1.95 \%$ \\
\hline 3 & 10 & 3 & $0.92 \%$ \\
\hline 3 & - & 9 & $2.77 \%$ \\
\hline
\end{tabular}

\begin{tabular}{|l|l|l|l|}
\hline \multicolumn{5}{|c|}{312 praetas de 4 pulebrus } \\
\hline 4 & 1 & 261 & $83.65 \%$ \\
\hline 4 & 2 & 21 & $8.73 \%$ \\
\hline 4 & 3 & 9 & $2.88 \%$ \\
\hline 4 & 4 & 5 & $1.60 \%$ \\
\hline 4 & 6 & 4 & $1.28 \%$ \\
\hline 4 & 6 & 1 & $0.32 \%$ \\
\hline 4 & 7 & 0 & $0.00 \%$ \\
\hline 4 & 8 & 1 & $0.32 \%$ \\
\hline 1 & 2 & 0 & $0.00 \%$ \\
\hline 4 & 10 & 1 & $0.32 \%$ \\
\hline 4 & - & 9 & $2.89 \%$ \\
\hline
\end{tabular}

Los resultados, como puede observarse, se han se pa ra do en cua tro ta blas. La pri me ra ta bla su ma riza los re sul ta dos de to das aquellas pe ti ciones de bús que da de una sola pa la bra, la se gun da tabla las pe ti cio nes con dos pa labras, la tercera con tres palabras y la cuarta con cuatro palabras. Cada una de estas cuatro tablas de resultados está conformada a su vez por once renglones. En el primer renglón de cada ta bla se mues tra el nú me ro (con su porcentaje correspondiente al total de pruebas por tabla) de documentos que fueron encontra dos en el pri mer lu gar de la lis ta or de na da. En el segundo renglón se muestra el número de documen tos que fue ron en con tra dos en la segunda posición dentro de la lista. El n-ésimo ren glón $(1=10)$ mues tra el nú me ro de docu men tos que fue ron en con tra dos en la n-ésima posición dentro de la lista ordenada. El úl ti mo ren glón mues tra el nú me ro de pe ti ciones de búsqueda fallidas.

Debe observarse que los resultados presentados en las ta blas an te rio res no re pre sen tan una eva lua ción de unSRI tal como se su gie re.(6 y 7) Sin em bar go, se con si de ra que es tas ta blas refle jan de ma ne ra muy apro xi ma da la pre ci sión -no el re call(6 y 7) - real del SRI ba sa do en triadas. Más aún, se tiene la certeza de que si un gru po de per so nas cons tru ye un pa drón de peti cio nes más cer ca no a la rea li dad y si ade más se emplean personas que analicen los documentos recuperados en cada bús que da del padrón, los re por tes de pre ci sión se rán me jo res.

Finalmente se comenta que estas pruebas de evaluación fueron realizadas en un equipo SUN 630/MP con el cual se encontró que el tiem po de res pues ta pro me dio de una bús queda sobre toda la colección (más de medio millón de fichas) es de alrededor de tres segundos.

\section{FU TUROS TRA BA JOS DE IN TERÉS}

\section{SO BRE UN TEMA}

Algunos puntos de los que sería interesante seguir investigando son:

a) Rea li zar otro tipo de prue bas de evaluación.(6 y 7)

b) Experimentar con otras formas de or gani zar las re pre sen ta ciones de los documentos, que probablementedarán mayor rapidez a la consulta de los documentos. Entre las técnicas más comunes a este respecto, se en- 
cuen tra la for ma ción de agru pa mien tos de representaciones. (6 y 7)

c) Proponer y experimentar con otras medidas de similitud.

d) Experimentar con otro tipo de indexación, por ejemplo indexación por cuartetas de letras.

e) Desarrollar una metodología más simple que las pruebas de evaluación, para de fi nir ellen gua je de in dexación.

\section{CONCLUSIONES}

Se han utilizado,hasta el momento, a los SRI por indexación de triadas en el desarrollo de sis te mas au to ma ti za dos de con sul ta de in formación, los cuales mane jan di ver sos ti pos de co lec ciones como son: ar tícu los de pe riódi co y revistas, fichas bibliográficas y bancos de in for ma ción so bre per so nal y pro yec tos en la UNAM (ARIES). Cabe mencionar que varios de estos sistemas (incluyendo al sistema de consulta de fichas bibliográficas que se evaluó en la sec ción 8 de este tra ba jo) pue den ser accesadospúblicamente a través de gopher a la direcciónelec trónica:

\section{info@condor.dgsca.unam.mx.}

La ex pe rien cia en el de sa rro llo de es tos sis temas, nos permite hacer las siguientes observa cio nes acer ca de los SRI por in de xa ción de triadas.

a) Rapidez en las búsquedas.

b) Los archivos de indexación no son demasiado grandes comparadoscon otros sistemas de consulta.

c) Este tipo de sistemas de consulta resultan simples y naturales en su empleo para un usuario final, ya que no es ne ce sario em plearoperadores booleanos para formular las búsquedas. d) Independencia de la plataforma de cómputo en que operen.

e) Cuando se sabe que lo que se busca está en alguna parte de un banco de in for ma ción y se cuen ta con al gu nas piestas para encontrarlo (por ejemplo, al gu nas pala bras del docu men to buscado), este tipo de sistemas permiten obtener excelentes resultados (tienen buena precisión). Por otro lado, estos sistemas también pueden ser usados, aunque no son tan buenos, en búsquedas de tipo estadístico, en donde normalmente lo que se desea recuperar son todos los documentos d eun tema en particular, sin importarcuáles docu men tos son más afines a la petición de búsqueda (no tienen tan buen recall).

\section{BIBLIO GRA FÍA.}

1) R.S. Boyer and J.S. Mo ore, A fast string search al go rithm, Comm. ACM, 20, 762- 772 (1977).

2) D.M. Sunday, A very fast substring search algorithm, Comm. ACM, 33, 132-142(1990)

3) P.D. Smith, Ex pe ri ments with a very fast subs tring search al go rithm, Soft ware-Practice and Experience, 21(10), 1065-1074, Oct. 1991.

4) U. Man ber and S. Wu, An Al go rithm for aproxxi ma te string mat ching with non uni form costs, Te chnical Re port TR-89-19, De par ta ment of Compu ter Scien ce, Uni ver sity of Ari zo na, Tuc son, Ari zo na, Sept. 1989.

5) S. Wu and U. Man ber, Agrep - a fst appro xi ma te pat tern.mat ching tool, In Proce edings of USENIX Win ter 1992 Te chni cal Cou fe ren ce, 153-162, San Fran cis co, CA, Jan 1992.

6) C.J. van Rijsbergen, Information Retrieval, Butterworth \& Co. Ltd., Second Edi tion, 1979.

7) Ge rald Sal ton, Mi chael J. Mc Gill, In tro duc tion to mo dern in for ma tion retrieval, McGraw-Hill.

8) R.W. Hamming \& Englewood Cliffs, Coding and information theory, Pren ti ce - Hall, 1980.

9) Ge rar do Vega H., So fía un sis te ma de re cu pe ra ción de In for ma ción por inde xación de tria das, Te sis de maes tría,UACPy P delCCHUNAM, 1994. 